\title{
Analysis of Unsteady Transonic Flow Fields by Means of the Colour Streak Schlieren Method
}

J. Ulrych

This article deals with a new approach to the investigation of unsteady transonic flow fields around aerodynamic models and in blade cascades using a schlieren method of flow visualisation. The principle and the application of the Colour Streak Schlieren Method (CSSM) are defined. The characteristic flow field features were observed and analysed around an oscillating NACA 0012 airfoil under the conditions of transonic free stream Mach number $\left(M_{\infty}=0.9\right)$, initial angle of attack $(\alpha=+4$ deg), one amplitude of oscillation $(\Delta \alpha= \pm 3 \mathrm{deg})$, and three frequencies of model oscillation $(f=1,15,30 \mathrm{~Hz})$. There is a description of the terminal shock wave hysteresis across the investigated area, which was revealed in particular cases. Application possibilities of CSSM and its further development are discussed.

Keywords: High-speed aerodynamics, experiment, flow field analysis, transonic flow fields, flow visualisation, Colour Streak Schlieren Method.

\section{Notation}

\begin{tabular}{|c|c|c|}
\hline$c$ & {$[\mathrm{~mm}]$} & chord \\
\hline$d$ & {$[\mathrm{~mm}]$} & distance between streaks \\
\hline$f$ & {$[\mathrm{~Hz}]$} & frequency of oscillation \\
\hline $\mathrm{M}_{\infty}$ & [1] & free stream Mach number \\
\hline$t$ & {$[\mathrm{~mm}]$} & streak thickness \\
\hline$\alpha$ & [deg] & angle of attack \\
\hline$\Delta \alpha$ & {$[\mathrm{deg}]$} & amplitude of oscillation \\
\hline$\delta$ & [deg] & streak deflection \\
\hline$\rho$ & {$\left[\mathrm{kg} / \mathrm{m}^{3}\right]$} & density \\
\hline Seq. & & video sequence \\
\hline
\end{tabular}

\section{Introduction}

Optical methods of flow visualisation are firmly connected with experimental research techniques in the field of high-speed aerodynamics. All the optical methods utilize the change of density $\rho$ of the fluid passing the test section of a wind tunnel. There are three basic methods - shadowgraph, schlieren, and interferometric - which provide images of the flow field around bodies in test sections. In recent decades many modifications of these methods have been developed in order to enhance the quality of aerodynamic research.

Although a great effort has been made to obtain quantitative information from optical records based on shadowgraph and schlieren methods, only the interferometric method remains an effective way to acquire this data.

Nevertheless, qualitative information from shadowgraph and schlieren pictures gives us a helpful insight into the real transonic flow fields. Moreover, video records enable us to observe the changes in the investigated area under unsteady conditions, e.g. in the case of oscillating models, changing free stream Mach number, angle of attack or the frequency of oscillation, etc.

The aim of this paper is to describe a new method of flow field analysis which can reveal some hardly observable phenomena in unsteady transonic flows, and can obtain some quantitative data from schlieren video sequences.

\section{Experimental devices and aerodynamic model}

The schlieren video sequences that are analysed in this article were taken in the test section of a continuous transonic wind tunnel for single airfoils. The dimensions of the test section were $0.14 \mathrm{~m} \times 0.32 \mathrm{~m}$.

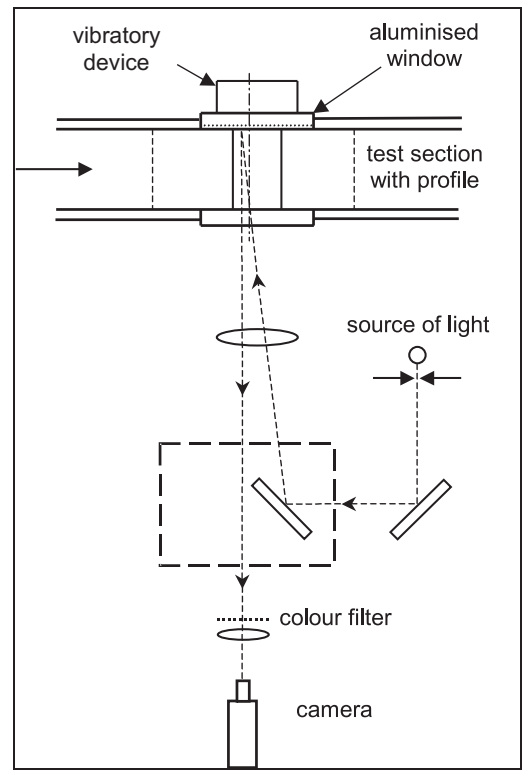

Fig. 1: Optical arrangement for schlieren method - oscillating profile configuration

All the records were obtained by means of a portable schlieren device, which is schematically depicted in Fig. 1. A halogen lamp was used as a source of light. The beam of rays was deflected by mirrors and then passed through a lens and an optical window into the test section. On the opposite wall of the wind tunnel the light was reflected from the aluminised surface of the optical window and passed the test section and lens once more. After passing through the colour filter the condensed rays were recorded by the high-speed camera. The camera operated at the frequency up to $2 \mathrm{kHz}$. 
A hydraulic crankshaft vibratory device was placed on the outer side of the tunnel behind the aluminised window. The apparatus was used to generate harmonic oscillation of the model within the range of frequencies $f=1-40 \mathrm{~Hz}$.

For the transonic flow field investigation around a body in the test section, the NACA 0012 airfoil was employed. The length of airfoil chord $c$ is $10 \mathrm{~cm}$.

\section{Optical measurement results}

The optical measurement results are represented by three movie sequences (Seq.1-3), which give us an idea about the flow field around a single NACA 0012 airfoil oscillating under transonic conditions [1], [2].

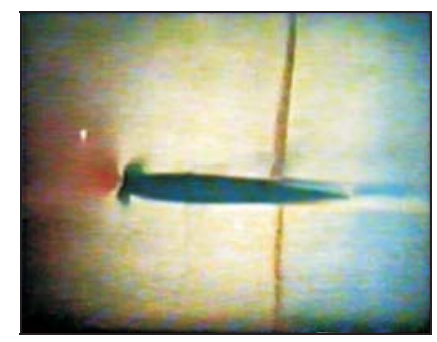

a)

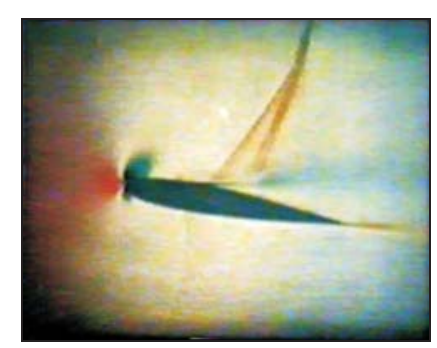

b)

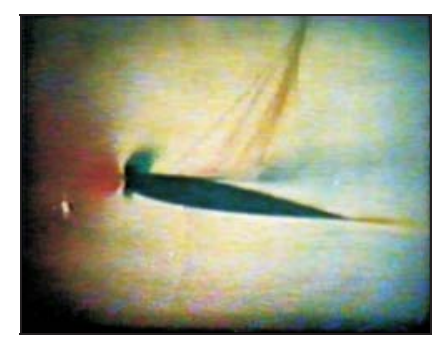

C)

Fig. 2: a) bottom dead centre, b) increasing $\alpha$, c) top dead centre

The initial angle of attack was set $\alpha=+4 \mathrm{deg}$, the amplitude of oscillation $\Delta \alpha= \pm 3 \mathrm{deg}$, and the Mach number of incoming flow $\mathbf{M}_{\infty}=0.9$. The frequency was arranged $f=1,15$, and $30 \mathrm{~Hz}$. All the records were taken with the frequency $1 \mathrm{kHz}$.

The first sequence introduces the situation characterised by the initial angle of attack $\alpha=+4 \mathrm{deg}$ and frequency $f=1 \mathrm{~Hz}$ we can to observe a red region of flow deceleration in the vicinity of the leading edge, a green region of acceleration, the compression region on the upper side of the profile, the interaction between the terminal shock wave and the boundary layer, and the separation point. Downstream, the separation point shear layer and wake are clearly visible.

The response of the flow field features to the unsteady boundary value conditions is represented by the oscillations of the separation point, shock wave, and shear layer. The boundaries of the deceleration and acceleration areas close to the leading edge also vary according to the current angle of attack.

At the bottom dead centre ( $\alpha=1 \mathrm{deg}$ ) the flow around the airfoil is nearly symmetric. The terminal shock wave on the upper side is situated at approximately $75 \%$ of the chord length and it is almost perpendicular to the chord. The separation point is at the place of interaction between the shock wave and the boundary layer. The situation on the lower side is similar, only the terminal shock wave is slightly bit nearer to the trailing edge. As the angle of attack increases the compression area on the upper side is more intensive and a $\lambda$-shock is formed. Close to the top dead centre the shock wave becomes less intensive and oscillates with significantly higher frequency.

The increase in the oscillation frequency $(f=15,30 \mathrm{~Hz})$ results again in the development of a $\lambda$-shock on the upper side (Fig. 2b) and the flow field features become more distinctive. Close to the top dead centre of the profile oscillation the shock wave starts to divide and again oscillate with significantly higher frequency.

Figs. 2a-c demonstrate the characteristic positions of the aerofoil in the case of Seq. 3 at $f=30 \mathrm{~Hz}$.

\section{Optical measurement analysis - Colour Streak Schlieren Method}

The Colour Streak Schlieren Method (CSSM) has been developed in order to examine the response of the flow around an aerodynamic model to unsteady boundary value conditions [1], [2]. The principle of the method is to observe the change of flow field phenomena position in time according to the change of the free stream conditions or the model oscillation.

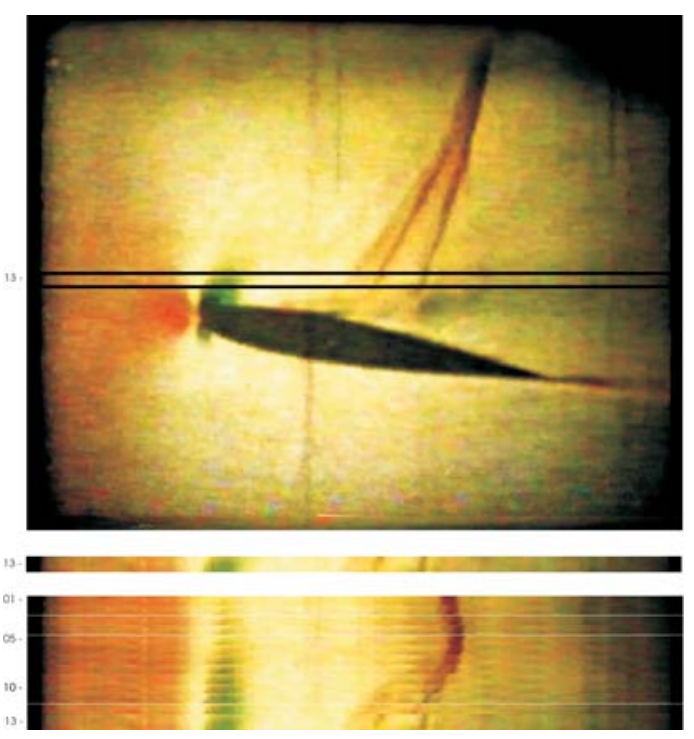

Fig. 3: Principle of the Colour Streak Schlieren Method

At the very beginning the area of interest (streak) in the investigated field has to be defined. The dimensions, position and deflection of the streak must be determined with respect to the intelligibility of the final picture. The deflection $\delta$ is 
used to place the streak in the required direction (e.g., when the streak must be parallel or perpendicular or oblique to the model or free stream). Then the selected part of the schlieren picture is extracted from each video frame and lined up according to time succession. The whole procedure is depicted in Fig. 3.

The time factor can be deduced from the harmonic oscillation of the model. This physical quantity can then be used for obtaining some quantitative information, for example, about the higher frequency of oscillation of the shock waves near to the maximum angle of attack.

Essentially, the method can be applied to every basic variant of optical visualisation mentioned above. However, the interferograms become unintelligible when the streaks are assembled. This problem does not occur when the shadowgraph method is employed. The disadvantage of the shadowgraph method lies in the lesser sensitivity to the change of fluid density around the body in the flow. Therefore, only the intensive density changes can be recorded and subsequently analysed. Colour schlieren video images are therefore the most suitable for such an analysis.

\section{Application of CSSM to an oscillating airfoil}

Development and testing of CSSM has been carried out using the schlieren video sequences of an oscillating airfoil, as described above.

The first step in the analysis was done to specify the major factors influencing the character and position of the flow field phenomena. In this particular case the following major factors were defined: free stream Mach number $\mathbf{M}_{\infty}$, angle of attack $\alpha$, amplitude of oscillation $\Delta \alpha$, frequency of oscillation $f$, and the position of the streak in the examined area defined by the $y$ coordinate and deflection angle $\delta$.

The streak thickness $t$ and the number of streaks also determine the final picture.

For the CSSM analysis $\mathrm{M}_{\infty}=0.9, \alpha=4 \mathrm{deg}, \Delta \alpha= \pm 3 \mathrm{deg}$, $f=1,15,30 \mathrm{~Hz}$, and $t=2.2 \mathrm{~mm}$. The y-axis started at the centre of model rotation; the $y$ coordinate of the first streak was chosen in all cases just above the upper side of the airfoil $\left(y_{1}=8 \mathrm{~mm}\right)$. The distance between the subsequent streaks was $d=9 \mathrm{~mm}$. The values of streak deflection were chosen $\delta=-0.5 \operatorname{deg}(f=1 \mathrm{~Hz}), 1.5 \operatorname{deg}(f=15 \mathrm{~Hz}), 2 \operatorname{deg}(f=30 \mathrm{~Hz})$ in order to eliminate the camera setting inaccuracy.

Fig. 4 introduces an example of the final flow field image after CSSM application. The picture corresponds to $\mathrm{M}_{\infty}=0.9$, $\alpha=4 \mathrm{deg}, \Delta \alpha= \pm 3 \mathrm{deg}, f=1 \mathrm{~Hz}, y=17 \mathrm{~mm}, t=2.2 \mathrm{~mm}$.

According to Fig. 2 the red area on the left relates to the deceleration of the flow upstream the leading edge, the green area represents the flow acceleration downstream the stagnation point, the compression region is grey, and the brown line defines the terminal shock wave. The decreasing intensity of the shock close to the top dead centre of airfoil oscillation is clearly visible - the brown line becomes lighter. (The technical reasons do not allow to print in colours.)

A comparison of the flows characterised by different oscillation frequencies is made in Figs. $5 \mathrm{a}-\mathrm{c}$. The pictures concentrate on one cycle; the streak related to the top dead centre ( $\alpha=7 \mathrm{deg}$ ) is first from the top, and the bottom dead centre

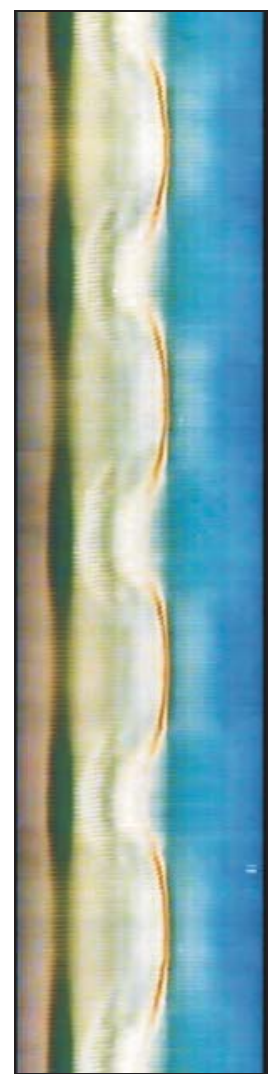

Fig. 4: CSSM - the final flow field image

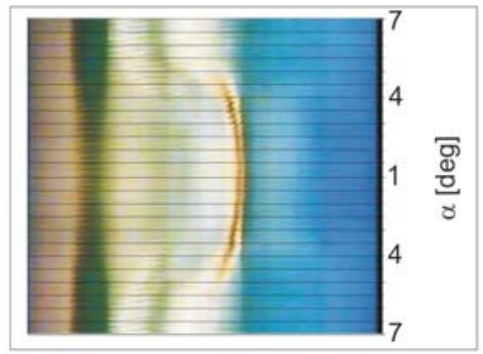

a)

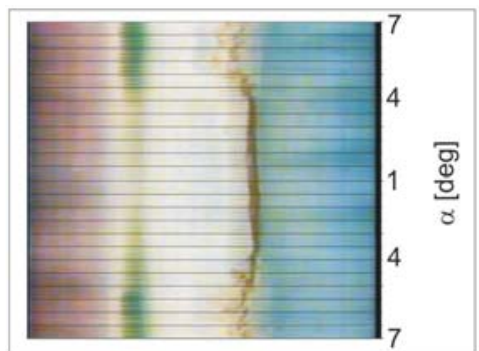

b)

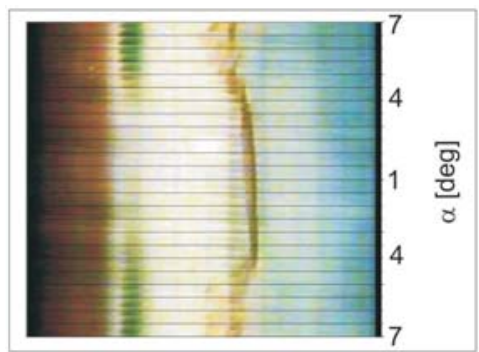

C) 
corresponds to the streak in the middle of the picture. All the characteristics are the same as in the case shown in Fig. 4 except the frequencies, with values are 1,15 , and $30 \mathrm{~Hz}$, respectively.

In the case of $f=1 \mathrm{~Hz}$ it is evident that the oscillations of the flow field features are harmonic and keep the same frequency. As the oscillation frequency increases the shock wave oscillation loses its harmonic character and hysteresis occurs in the vicinity of the middle angle of attack ( $\alpha=4 \mathrm{deg}$ ). The comparison of the shock wave position in time and the harmonic oscillation of the model shows no phase shift. Shock wave splitting can be observed near to the top dead centres and the subsequent increase in the oscillation frequency of individual branches.

A better analysis of the shock wave hysteresis was done for the oscillation frequency $f=30 \mathrm{~Hz}$, where the effect is most noticeable. The process is depicted in Fig. 6. The flow field was examined in six streak positions. Streak No. 1 is situated approximately $8 \mathrm{~mm}$ above the airfoil centre of rotation, and the next streaks are placed at a distance 17, 26, 35, 44, and $53 \mathrm{~mm}$.

It is obvious from the picture introducing one cycle that the hysteresis has its maximum value around $\alpha=3.5 \mathrm{deg}$. Close to the upper side of the airfoil the difference between the shock wave positions during the increase and decrease of $\alpha$ is almost $1 \mathrm{~cm}$ (approximately $10 \%$ of a chord length) and going further away from the surface this difference is even greater. This effect can be visualised using a combination of two photographs in Fig. 7. The first image is related to the decreasing angle of attack in the position $\alpha=3.5 \mathrm{deg}$ (the first shock wave from the left), while the latter is related to the increasing angle of attack in the same position. The difference between the shock wave locations is clearly visible. Due to the velocity distribution around the airfoil the effective angle of attack seems to be bigger than $\alpha=3.5 \mathrm{deg}$ when the leading edge is moving downward. Subsequently the separation point shifts upstream (two red points in Fig. 7) and so does the terminal shock wave. During the upward move of the leading edge the situation is converse. Following the black lines along the shock waves from the airfoil it can be seen that the distance between shocks becomes smaller close to the surface. Then the difference increases again. The actual shape of a sonic region around the airfoil causes this curvature.

Fig. 8 shows a CSSM picture of 5 cycles recorded in the same six positions as in Fig. 6 . The terminal shock oscillation becomes less harmonic as the distance from the airfoil increases. At a distance of approximately one half of the chord length from the airfoil centre of rotation it is practically impossible to distinguish the streaks related to the dead centres of model oscillation.

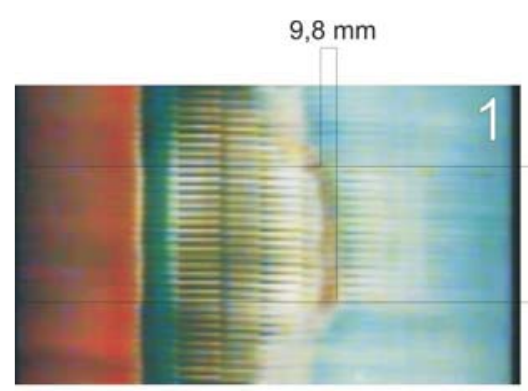

$9,8 \mathrm{~mm}$
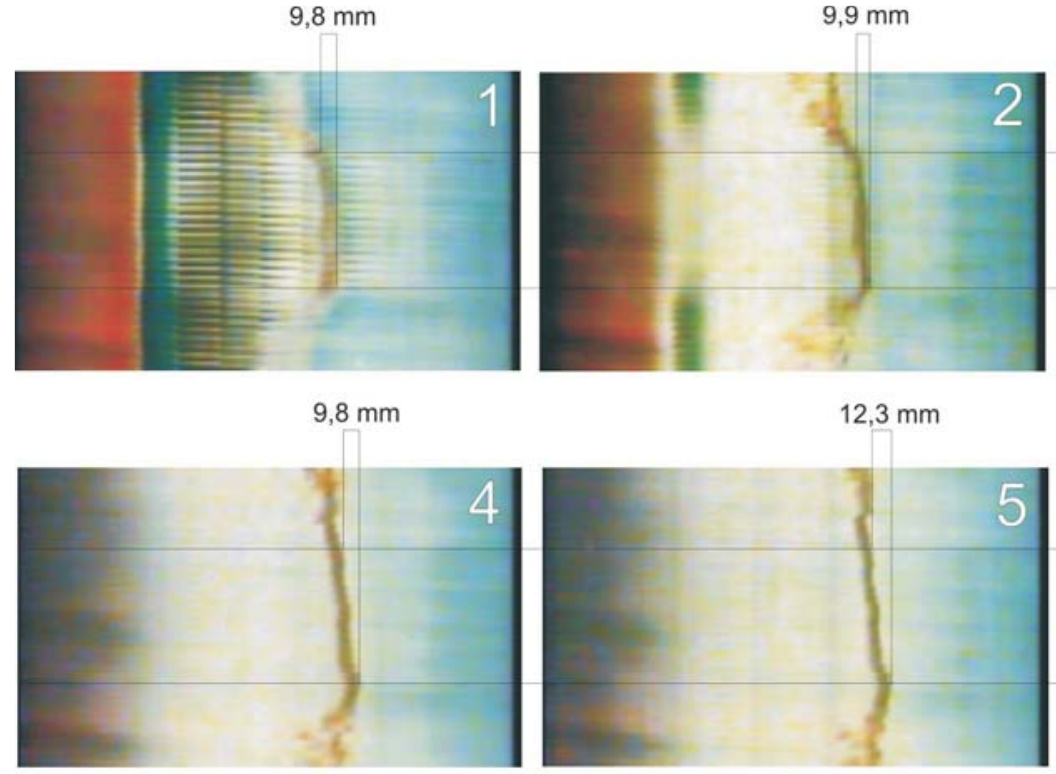

2

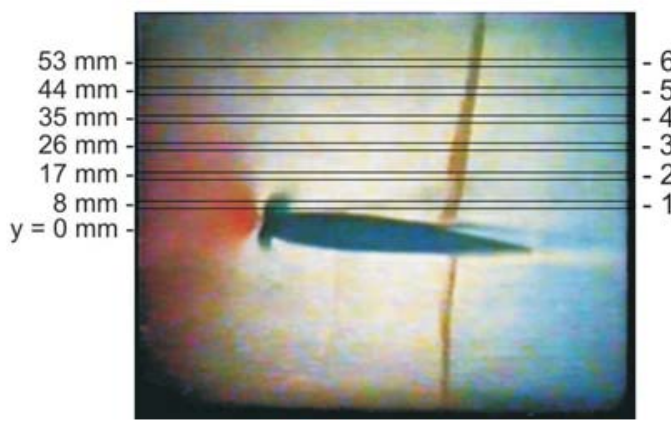

Fig. 6: Terminal shock wave hysteresis
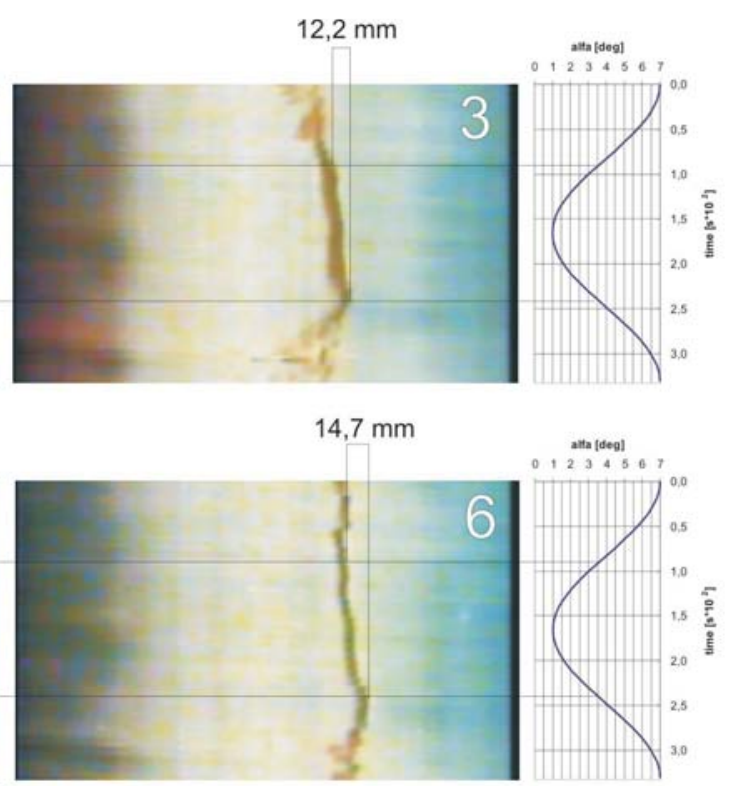


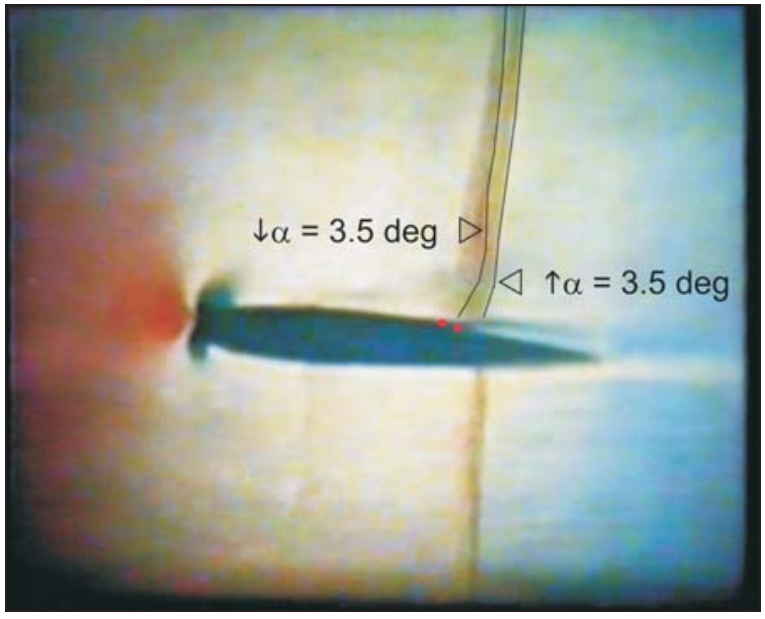

Fig. 7: Shock wave hysteresis, $\alpha=3.5 \mathrm{deg}$

\section{Conclusions}

The Colour Streak Schlieren Method represents a new approach to flow field analysis based on optical records. It can be used as an independent method for evaluation of experimental and CFD research in the field of high-speed aerodynamics. Basically it utilizes video sequences taken by means of the
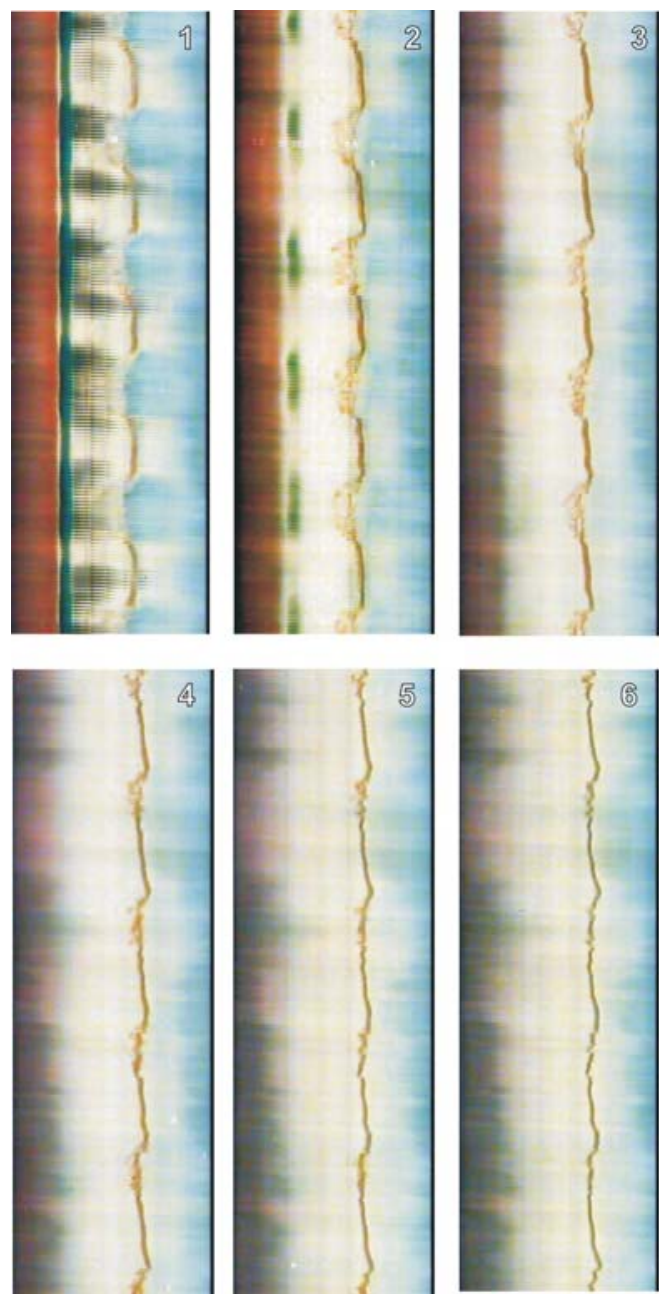

Fig. 8: CSSM pictures of terminal shock wave oscillation above the NACA 0012 airfoil schlieren method. Applications to shadowgraph and interferometric methods are possible, but the results are not of the same quality.

CSSM has been developed for the investigation of unsteady high subsonic, transonic, and supersonic flow fields around the bodies or in blade channels in test sections of wind tunnels. The method enables hardly observable phenomena to be revealed, and their time dependence to be examined. Some quantitative information related to the position of flow field features can be obtained.

The possibilities of CSSM were demonstrated in an nalysis of the flow around an oscillating airfoil under transonic conditions. The shock wave hysteresis was disclosed, described, and quantified. The results meet the results reported in [3].

Further development of the method will focus on the application of Fourier analysis to the final CSSM pictures. This improvement could be of significant importance for the investigation of phenomena like oscillations of shock waves with higher frequency described in this paper, or the transonic instability that has been recorded in blade cascades [1].

\section{Acknowledgments}

All the experiments were carried out at the Department of High-Speed Aerodynamics, Aeronautical Research and Test Institute, Prague. The electronic data processing was accomplished in cooperation with the Department of Aerospace Engineering, Czech Technical University in Prague. The support for this research from the Aerospace Research Centre is gratefully acknowledged.

\section{REFERENCES}

[1] Ulrych J., Benetka J., Šafařík P.: "Records of Unsteady Transonic Flow Past Blade Cascades by Means of Optical Methods". In: Measuring Techniques in Turbomachinery, Proceedings of the $16^{\text {th }}$ Symposium on Measuring Techniques for Transonic and Supersonic Flow in Cascades and Turbomachines, Cambridge, 2002, to be printed in 2003.

[2] Ulrych J., Benetka J.: "Records of Unsteady Transonic Flow by Means of Schlieren Video Images". In: Fluid Dynamics 2002, Proceedings of the Colloquium at Institute of Thermomechanics, Academy of Sciences of the Czech Republic, Prague, 2002, p. 179-182.

[3] Benetka J., Pernica Z.: "Harmonically Oscillating Airfoil in Transonic Stream of Air". ARTI Report, Z-34, Prague, 1980 .

Ing. Jiří Ulrych

phone/fax: +420284825347

e-mail: ulrych@aerospace.fsik.cvut.cz

Department of High-Speed Aerodynamics

Aeronautical Research and Test Institute Beranových 130

19905 Prague - Letňany, Czech Republic 